\title{
VARIAÇÃO DA DENSIDADE APARENTE E RESISTÊNCIA À COMPRESSÃO PARALELA ÀS FIBRAS EM FUNÇÃO DA INTENSIDADE DE DESBASTE, ADUBAÇÃO E POSIÇÃO RADIAL EM Eucalyptus grandis HILL EX-MAIDEN ${ }^{1}$
}

\author{
Israel Luiz de Lima² e José Nivaldo Garcia ${ }^{3}$
}

\begin{abstract}
RESUMO - O Eucalyptus grandis destaca-se pela produtividade e qualidade de sua madeira. O manejo florestal ideal das árvores em que se obtém maior proporção de madeira e melhor qualidade é uma das questões a serem consideradas nas pesquisas de E. grandis. Este trabalho teve como objetivo estudar a variação da densidade aparente e da resistência à compressão paralela às fibras em função da intensidade de desbaste, adubação e classe de diâmetro, na posição radial nas árvores de uma população de E. grandis de 21 anos de idade, manejada pelo sistema de desbastes seletivos com aplicação de fertilizantes na época do início dos desbastes, ou seja, aos 5 anos. Os fatores utilizados foram: três intensidades de desbastes seletivos (37, 50 e 75\%), presença ou ausência de fertilizantes, três classes de diâmetro e cinco posições radiais. As influências dos fatores e de suas combinações foram avaliadas na densidade aparente e na resistência à compressão da madeira. A densidade aparente da madeira e a resistência à compressão foram influenciadas pelos fatores: adubo e classe de diâmetro em quase todas as posições radiais aumentaram no sentido da medula para a casca. Observou-se relação positiva entre densidade aparente, resistência à compressão e posição radial.
\end{abstract}

Palavras-chave: Eucalyptus, Manejo e Propriedades da madeira.

\section{VARIATION OF THE SPECIFIC GRAVITY MASS AND THE COMPRESSION STRENGTH OF THE THINNING INTENSITY, FERTILIZATION AND THE RADIAL POSITION IN Eucalyptus grandis HILL EX-MAIDEN}

\begin{abstract}
The Eucalyptu grandis stands apart for its productivity and quality wood. The ideal forest management where a greater wood ratio and better quality are obtained is one of the questions to be considered in the research of the $\boldsymbol{E}$. grandis. The present work had as its general objective the study of the variation of the specific gravity and the resistance to the compression as a function of the thinning intensity, fertilization and diameter classes in the radial position in trees of a 21-year old population of $\boldsymbol{E}$. grandis, managed under the system of selective thinning, with the application of fertilizers. The factors used in this study were: three intensities of selective thinning (37, 50 and 75\%), presence or absence of fertilizers, three diameter classes and five radial positions. The influences of the factors and of their combinations were evaluated regarding specific gravity and compression strength. The specific gravity and compression strength of the wood were influenced by factors such as fertilizer and diameter class in almost all radial positions, increasing significantly from the pith to bark. A good positive relationship was found to exist among the specific mass, compression strength and radial position.
\end{abstract}

Keywords: Eucalyptus, Management and Wood properties.

\section{INTRODUÇÃO}

O gênero Eucalyptus apresenta grande potencial para processamento mecânico da madeira, por ser de rápido crescimento, possuir boa adaptação às condições ambientais encontradas em grande parte do Brasil e por sua madeira apresentar boas propriedades físicas e mecânicas e ser de fácil trabalhabilidade, além de poder ser produzida a baixo custo. Há ainda no gênero grande variedade de cores

\footnotetext{
${ }^{1}$ Recebido em 05.10.2007 e aceito para publicação em 02.03.2010.

${ }^{2}$ Instituto Florestal do Estado de São Paulo, IF, Brasil. E-mail: <israelluizde.lima@yahoo.com.br>.

3 Universidade de São Paulo, USP, Brasil.. E-mail: <jngarcia@esalq.usp.br>
} 
que contribui para destacar e valorizar o seu grande potencial de substituição de espécies tradicionalmente utilizadas em certos usos.

De modo geral, podem-se melhorar, modificar, controlar ou minimizar os fatores que afetam a quantidade e qualidade da madeira, por meio de tratos silviculturais. Entre os tratos silviculturais que possibilitam alterações nas condições e taxas de crescimento das árvores, destacam-se a aplicação de fertilizantes e os desbastes que podem ser utilizados para aumentar a quantidade e melhorar a qualidade da madeira produzida.

De acordo com Harris (1981), tanto em coníferas quanto em folhosas o crescimento mais rápido da população, devido às alterações nas condições de crescimento, pode ocasionar a diminuição do comprimento da fibra, da densidade aparente da madeira e da resistência, além de aumentar seu gradiente na direção medula-casca.

Um dos grandes desafios da produção de madeira de reflorestamento é minimizar a formação de madeira juvenil em árvores jovens, de crescimento rápido e de pequenas dimensões que, inevitavelmente, tendem a compor o mercado madeireiro num futuro bastante próximo.

Segundo Malan (1995), a prática de desbastes causa efeito no desenvolvimento da copa e na taxa de crescimento das árvores, podendo causar alteração significativa na formação da madeira. Isso pode resultar em aumento da área de madeira juvenil, produzindo madeira de baixa densidade e fibras curtas, além de alta retração longitudinal sob secagem e do alto teor relativo de lignina.

Vital (1990) destacou, citando vários autores, a existência de resultados conflitantes sobre o efeito da fertilização na densidade da madeira, descrevendo trabalhos em que por vezes, obtivera redução, por outras vezes aumento ou não alteração nas propriedades com a adubação, mas enfatizando que, se houver diminuição, o peso de madeira produzida por hectare será maior devido ao grande crescimento volumétrico.

Andrade et al. (1994), estudando o efeito da fertilização na qualidade da madeira de E. grandis aos 6 anos de idade, observaram que na densidade não houve diferenças significativas, porém ocorreu ligeira diminuição em relação às árvores não adubadas. A produção de massa foi afetada pela fertilização, havendo acréscimo significativo desse parâmetro em decorrência do aumento da produção volumétrica, visto que a densidade não foi afetada significativamente.

Wilkins e Horne (1991), trabalhando com população de E. grandis de 9,5 anos de idade, estudaram o efeito de vários tratamentos silviculturais, sobre a densidade básica da madeira. Eles verificaram que no tratamento em que ocorreram desbaste, fertilização e sulcamento, foram obtidos os maiores valores da densidade básica que também houve alteração na distribuição dos valores na árvore.

Berger et al. (2000) estudaram o efeito do espaçamento ( $3 \times 2$, $3 \times 3$ e $3 \times 4$ m) e da adubação (400 e $800 \mathrm{~kg} \mathrm{ha}^{-1}$ de NPK) sobre a Densidade Básica (DB), Módulo de Elasticidade (MOE) e Módulo de Ruptura (MOR) na população de um clone de E. saligna aos 10 anos de idade. Concluíram que os fatores adubação e espaçamento tiveram efeitos significativos sobre a variável densidade básica, ressaltando que a maior dose de adubo e o maior espaçamento proporcionaram aumento significativo dessa propriedade. Nos estudos de resistência à flexão estática, o MOE e o MOR foram significativamente influenciados pelo espaçamento, adubação e posição da tora. A maior dose de adubo e o maior espaçamento proporcionaram os maiores valores médios de MOE e MOR, diferindo significativamente da menor dose de adubo (400 $\mathrm{kg} \mathrm{ha}^{-1}$ ) e dos menores espaçamentos ( $3 \times 2$ e $3 \times 3 \mathrm{~m}$ ).

Lima e Garcia (2005) concluíram, trabalhando com E. grandis de 18 anos, que diferentes intensidades de desbaste não influenciaram significativamente as seguintes propriedades: densidade aparente, retração volumétrica e resistência à compressão paralela às fibras, entretanto, com relação à densidade básica, foi constatada diferença entre as intensidades de 67\% e 75\%.

Cruz et al. (2003), em estudo sobre variações de propriedades físicas e mecânicas da madeira de clones de híbridos de Eucalyptus, encontraram que tanto as propriedades físicas quanto as mecânicas apresentaram maiores variações na direção da medula para a casca do que da base para o topo das árvores, mostrando a importância do conhecimento das variações radiais nas toras para classificação da madeira serrada e, conseqüentemente para homogeneização.

Polli et al. (2006) observaram em populações clonais de $E$. grandis, que a densidade da madeira aumentou significativamente no sentido medula-casca e decresceu no sentido base-topo. A densidade da madeira próxima 
à casca $\left(0,57 \mathrm{~g} \mathrm{~cm}^{-3}\right)$ foi significativamente mais elevada do que na posição intermediária $\left(0,52 \mathrm{~g} \mathrm{~cm}^{-3}\right)$ e próxima à medula $\left(0,51 \mathrm{~g} \mathrm{~cm}^{-3}\right)$.

O objetivo deste trabalho foi estudar a variação da densidade aparente e da resistência à compressão paralela às fibras em função da intensidade de desbaste, adubação, classes de diâmetro e posição radial, em árvores de população plantada de Eucalyptus grandis de 21 anos de idade.

\section{MATERIAL E MÉTODOS}

O material utilizado nesta pesquisa foi obtido de população experimental de Eucalyptus grandis Hill ex-Maiden, de 21 anos de idade, localizada no Município de Lençóis Paulista, SP. O solo local foi classificado como Latossolo Vermelho Amarelo, de textura média e o clima é do tipo CWa, segundo a classificação de Köppen.

O ensaio foi composto por seis parcelas de $900 \mathrm{~m}^{2}$ cada uma, representando tratamento diferente. As árvores foram plantadas em 16/12/1982 em espaçamento de 3,0 x 1,5 m, totalizando 2.222 árvores ha-1 ${ }^{-1}$ Inicialmente foram aplicados $222 \mathrm{~kg} \mathrm{ha}^{-1}$ de NPK 6:30:6, distribuídos nos sulcos de plantio. Em 1987 foram iniciados desbastes do tipo seletivo, nas intensidades de 37, 50 e 75\%. Após o desbaste foi feita a adubação com $1.000 \mathrm{~kg}$ de NPK 12:06:12 e $100 \mathrm{~kg}$ de Lama-cal por hectare, distribuídos nas entrelinhas de apenas três das parcelas, caracterizando, portanto, tratamentos com e sem adubo.

Em seguida à adubação, foi efetuada gradagem leve para incorporar o adubo ao solo. Após o último dos três desbastes sem negrito que ocorreu aos 8,5 anos, o experimento foi mantido sem alteração até o início deste presente estudo. A Tabela 1 mostra os tratamentos da população experimental que foram selecionados para serem estudados.
Inicialmente, realizou-se um inventário do experimento e a partir da distribuição diamétrica obtida, foram eleitas três classes de diâmetro à altura do peito (DAP) para o estudo, ou seja: a classe inferior (DAP entre 20 e $25 \mathrm{~cm}$ ), classe mediana (DAP entre 25 e $30 \mathrm{~cm}$ ) e classe superior (DAP entre 30 e $35 \mathrm{~cm}$ ).

Dentro de cada parcela foram tomadas 12 árvores, sendo quatro em cada classe definidas, totalizando 72 árvores, que foram devidamente identificadas no campo. Na base de cada árvore selecionada, foi retirada uma tora de 1,70 m de comprimento.

As toras foram transportadas para a serraria da Escola Superior de Agricultura Luiz de Queiroz (ESALQ/ USP), em Piracicaba, SP, e desdobradas numa serra de fita simples, segundo o esquema mostrado na Figura 1 , de forma a produzir uma prancha diametral passível de fornecer corpos-de-prova representativos das posições 0, 25, 50, 75 e 100\% do raio da árvore, necessário ao estudo da variação medula-casca de forma completa. Essa técnica de desdobro foi adotada para evitar a rachadura que normalmente ocorre ao longo da medula da prancha diametral, por influência das tensões residuais remanescentes na tora. O primeiro corte teve o papel importante de liberar uma parcela da componente longitudinal da tensão de crescimento permitindo, que parte da energia armazenada fosse dissipada na forma de encurvamentos das duas peças produzidas. Esse alívio de tensões provocou suavização do gradiente de tensões ao longo do diâmetro perpendicular a esse corte a ponto de resultar em valores insuficientes para provocar a rachadura de medula muito comum em pranchas diametrais completas.

A prancha central, ainda no estado verde, foi subdividida através de cortes transversais em quatro peças de $43 \mathrm{~cm}$ de comprimento, e de cada uma dessas peças foram obtidos, através de cortes longitudinais,

Tabela 1 - Tratamentos selecionados dentro de uma população experimental de E. grandis de 21 anos de idade.

Table 1 - Treatments selected from an experimental population of 21 year-old E. grandis.

\begin{tabular}{|c|c|c|c|c|c|c|}
\hline Parcela & $\begin{array}{l}\text { adubação } \\
\text { aos5 anos }\end{array}$ & (\%) desbaste & $\begin{array}{c}\text { idade no } \\
\text { 1o desbaste }^{\text {id }}\end{array}$ & 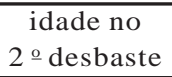 & $\begin{array}{c}\text { Idade no } \\
3 \stackrel{\circ}{\text { odesbaste }}\end{array}$ & $\begin{array}{c}\mathrm{N}^{\mathrm{o}} \text { de árvores } \mathrm{ha}^{-1} \\
\text { aos } 21 \text { anos }\end{array}$ \\
\hline 1 & $\mathrm{~N}$ & $37 \%$ & 5 & 6,5 & 8,5 & 549 \\
\hline 2 & S & $37 \%$ & 5 & 6,5 & 8,5 & 521 \\
\hline 3 & $\mathrm{~N}$ & $50 \%$ & 5 & 7,5 & - & 535 \\
\hline 4 & S & $50 \%$ & 5 & 7,5 & - & 556 \\
\hline 5 & $\mathrm{~N}$ & $75 \%$ & 5 & - & - & 535 \\
\hline 6 & S & $75 \%$ & 5 & - & - & 535 \\
\hline
\end{tabular}

OBS.: $\mathrm{N}$ = não adubada; $\mathrm{S}$ = adubada.

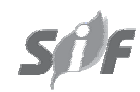

Revista Árvore, Viçosa-MG, v.34, n.3, p.551-559, 2010 


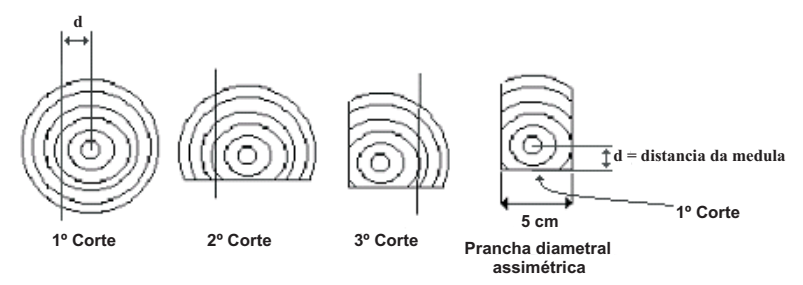

Figura 1 - Sequência de cortes para obtenção da prancha diametral isenta da rachadura longitudinal de medula.

Figure 1 - Sequence of cuts in order to obtain the diametrical board exempt from the longitudinal crack of pith.

dois ou três sarrafos de (4 x 4 x $43 \mathrm{~cm})$, segundo metodologia mostrada na Figura 2. Esse número variável de sarrafos por peça foi adotado para que fosse possível a obtenção de dois sarrafos em cada uma das posições radiais de estudo, principalmente no caso das pranchas de pequena largura obtidas de árvores de diâmetro da classe inferior.

Os sarrafos foram secos ao ar por um período de quatro meses, sendo, então, selecionado aleatoriamente um dos dois sarrafos de cada posição radial para casualizar a amostragem ao longo da altura da tora. Preferiu-se, assim, confundir o possível efeito da posição longitudinal com o erro experimental. Foi obtido desse sarrafo um corpo-de-prova para o ensaio de compressão e outro para o ensaio de densidade aparente, totalizando 720

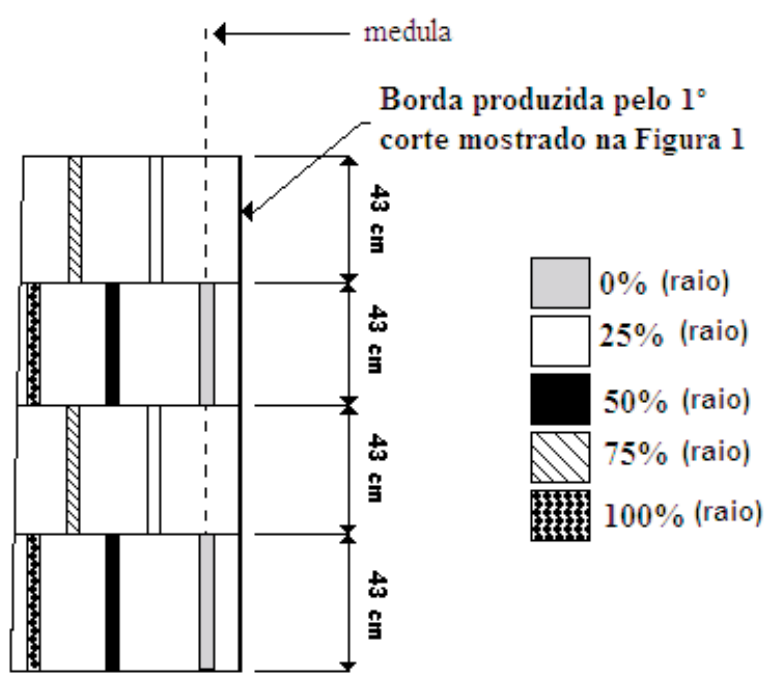

Figura 2 - Prancha central representando as posições radiais de retirada dos corpos-de-prova para estudo da variabilidade radial.

Figure 2-Central board representing the radial positions of specimens removal for radial variability study. corpos-de-prova, sendo 360 para estudo da variabilidade da densidade aparente e 360 para resistência à compressão paralela às fibras na umidade de equilíbrio ao ar (12\%), em função dos tratamentos adotados.

Para o estudo da densidade aparente foram confeccionados corpos-de-prova de pequenas dimensões ( 3 x 2,5 x 2,5 cm), menores do que o estabelecido pela norma (ABNT, 1997), devido ao fato de o objetivo principal deste trabalho ser o estudo da variação radial e não a caracterização dessa propriedade. A densidade aparente foi determinada através da expressão (1), obtendo-se a massa do corpo-de-prova em uma balança digital e obtendo as medidas das arestas do corpode-prova com um paquímetro digital.

$$
\text { em que: } \quad \rho_{a p}=\frac{\boldsymbol{m}_{12}}{\boldsymbol{V}_{12}}
$$

$\rho_{a p}$ : densidade aparente $\left(\mathrm{g} \mathrm{cm}^{-3}\right)$;

$m_{12}$ : massa da madeira a $12 \%$ de umidade (g) e

$V_{12}$ : é o volume da madeira a $12 \%\left(\mathrm{~cm}^{3}\right)$.

Pela mesma razão da densidade aparente, para se obter a resistência à compressão paralela às fibras, foram utilizados corpos-de-prova de pequenas dimensões (3 x 2,5 x 2,5 cm). Primeiramente foram medidas com um paquímetro digital, as áreas dos corpos-de-prova com teor de umidade em torno de $12 \%$, sendo em seguida realizado o teste de compressão paralela às fibras, em Máquina Universal de Ensaio com velocidade de aplicação de carga de 4.000 N min-1 (ABNT, 1997).

Para essa variável foi utilizada a expressão (2).

Em que:

$$
f_{c 0}=\frac{P_{r}}{A}
$$
$(\mathrm{MPa})$

$f_{\mathrm{c} 0}$ : resistência na compressão paralela às fibras

$$
P_{\mathrm{r}} \text { : carga de ruptura }(\mathrm{N}) \text { e; }
$$

$A$ : área da seção transversal $\left(\mathrm{mm}^{2}\right)$.

Para avaliação das variáveis, inicialmente foi efetuado o teste de homogeneidade de variância, por meio do teste de Hartley. Posteriormente, foi feito o teste $\mathrm{F}$, de análise de variância, segundo o delineamento experimental inteiramente casualizado, adotando-se o esquema fatorial 3 × 2 × 3 (desbastes $\mathrm{x}$ adubo $\mathrm{x}$ classes de DAP) para cada posição radial em separado. 
Foi aplicado o teste de Tukey a 5\% de significância sempre que observadas diferenças significativas em algum tratamento pelo teste F.

Estudou-se também a relação entre $f_{\mathrm{c} 0}, \rho_{a p}$ e posição radial, e para isso foi utilizada a análise de regressão, no nível de significância de 5\%.

Para análise dos dados foi utilizado o procedimento PROC GLM do programa estatístico SAS (SAS, 1999).

\section{RESULTADOS E DISCUSSÃO}

Nas Tabelas 2 e 3 são apresentados os resumos dos testes F das análises de variância da densidade aparente e resistência à compressão paralela às fibras. Nas Figuras 3 e 4, tem-se a representação das médias dos tratamentos, de todas as posições radiais.

Depreende-se, pelos resultados, obtidos, que a densidade aparente e a resistência à compressão não foram influenciadas significativamente pelas diferentes intensidades de desbaste aplicadas, quando avaliada a população com 21 anos de idade. Mesma tendência foi observada por Lima e Garcia (2005) em E. grandis com 18 anos de idade. Também não se verificou tendência sistemática da densidade aparente e da resistência à compressão em elevar os seus valores proporcionalmente com o aumento da intensidade de desbaste, conforme constatado por Land et al. (1983) e Malan e Hoon (1992). Entretanto, esses resultados podem ser considerados interessantes no sentido de ser possível aumentar o volume de madeira por área por meio da prática de desbaste, sem prejuízo significativo da densidade aparente e da resistência à compressão da madeira.

Quanto ao fator adubo, foi verificado que a densidade aparente e a resistência à compressão paralela às fibras das amostras oriundas das árvores das parcelas adubadas diferiram significativamente daquelas provenientes das parcelas não adubadas. Isso se deu em quase todas as posições radiais, com exceção da densidade nas

Tabela 2 - Valores de F obtidos nas análises de variância da $\rho_{a p}$ de $E$. grandis aos 21 anos.

Table 2 - Values of F obtained from the analysis of variance of $\rho_{a p}$ in the the 21-year-old E. grandis.

\begin{tabular}{|c|c|c|c|c|c|c|c|c|c|c|c|}
\hline \multirow[t]{2}{*}{ Causa de variação } & \multirow[t]{2}{*}{ GL } & \multicolumn{2}{|c|}{$0 \%$ (raio) } & \multicolumn{2}{|c|}{ 25\% (raio) } & \multicolumn{2}{|c|}{$50 \%$ (raio) } & \multicolumn{2}{|c|}{ 75\% (raio) } & \multicolumn{2}{|c|}{$100 \%$ (raio) } \\
\hline & & $\mathrm{F}$ & $\operatorname{Pr}>F$ & $\mathrm{~F}$ & $\operatorname{Pr}>\mathrm{F}$ & $\mathrm{F}$ & $\operatorname{Pr}>F$ & $\mathrm{~F}$ & $\operatorname{Pr}>F$ & $\mathrm{~F}$ & $\operatorname{Pr}>F$ \\
\hline Desbaste (D) & 2 & $0,51^{\text {n.s }}$ & 0,60 & $0,10^{\mathrm{n} . \mathrm{s}}$ & 0,90 & $0,41^{\text {n.s }}$ & 0,66 & $0,09^{\text {n.s }}$ & 0,91 & $0,13^{\text {n.s }}$ & 0,88 \\
\hline Adubo (A) & 1 & $12,05^{* *}$ & 0,00 & $8,53^{* *}$ & 0,00 & $15,61^{* *}$ & 0,00 & $3,87^{\text {n.s }}$ & 0,05 & $0,12^{\text {n.s. }}$ & 0,73 \\
\hline Classe (C) & 2 & $1,33^{\text {n.s }}$ & 0,27 & $3,45^{*}$ & 0,04 & $1,10^{\text {n.s }}$ & 0,34 & $4,12^{*}$ & 0,02 & $0,03^{n . s}$ & 0,97 \\
\hline $\mathrm{D} \times \mathrm{A}$ & 2 & $2,54^{\mathrm{n} . \mathrm{s}}$ & 0,09 & $0,62^{\text {n.s }}$ & 0,54 & $0,05^{\text {n.s }}$ & 0,95 & $0,28^{\text {n.s }}$ & 0,75 & $0,03^{n . s}$ & 0,97 \\
\hline $\mathrm{D} \times \mathrm{C}$ & 4 & $0,14^{\mathrm{n} . \mathrm{s}}$ & 0,97 & $1,94^{\mathrm{n} . \mathrm{s}}$ & 0,12 & $2,11^{\text {n.s }}$ & 0,09 & $2,71^{* *}$ & 0,03 & $0,95^{\text {n.s }}$ & 0,44 \\
\hline$A \times C$ & 2 & $1,11^{\mathrm{n} . \mathrm{s}}$ & 0,34 & $0,46^{\mathrm{n} . \mathrm{s}}$ & 0,63 & $1,01^{\text {n.s }}$ & 0,37 & $0,91^{\text {n.s }}$ & 0,40 & $1,76^{\mathrm{n} \cdot \mathrm{s}}$ & 0,18 \\
\hline $\mathrm{D} \times \mathrm{A} \times \mathrm{C}$ & 4 & $1,79^{\text {n.s }}$ & 0,14 & $1,63^{\mathrm{n} . \mathrm{s}}$ & 0,17 & $1,25^{\text {n.s }}$ & 0,30 & $0,27^{\mathrm{n} . \mathrm{s}}$ & 0,89 & $1,86^{\mathrm{n} \cdot \mathrm{s}}$ & 0,13 \\
\hline Resíduo & 54 & & & & & & & & & & \\
\hline
\end{tabular}

Em que: ns = não significativo a 5\% de significância; * = significativo a 5\% de significância; e ** = significativo a $1 \%$ de significância.

Tabela 3 - Valores de F obtidos nas análises de variância da $f_{\mathrm{c} 0}$ de E. grandis aos 21 anos.

Table 3 -Values of F obtained from the analysis of variance of $f_{\mathrm{c} 0}$ in the the 21-year-old E. grandis.

\begin{tabular}{|c|c|c|c|c|c|c|c|c|c|c|c|}
\hline \multirow[t]{2}{*}{ Causa de variação } & \multirow[t]{2}{*}{ GL } & \multicolumn{2}{|c|}{$0 \%$ (raio) } & \multicolumn{2}{|c|}{ 25\% (raio) } & \multicolumn{2}{|c|}{ 50\% (raio) } & \multicolumn{2}{|c|}{ 75\% (raio) } & \multicolumn{2}{|c|}{ 100\% (raio) } \\
\hline & & $\mathrm{F}$ & $\operatorname{Pr}>F$ & $\mathrm{~F}$ & $\overline{\operatorname{Pr}>F}$ & $\mathrm{~F}$ & $\operatorname{Pr}>F$ & $\mathrm{~F}$ & $\operatorname{Pr}>F$ & $\mathrm{~F}$ & $\operatorname{Pr}>F$ \\
\hline Desbaste (D) & 2 & $0,02^{\text {n.s }}$ & 0,98 & $1,82^{\text {n.s }}$ & 0,17 & $0,98^{\text {n.s }}$ & 0,38 & $0,09^{\text {n.s }}$ & 0,91 & $0,73^{n \cdot s}$ & 0,48 \\
\hline Adubo (A) & 1 & $10,07^{* *}$ & 0,00 & $10,87^{* *}$ & 0,00 & $11,50^{* *}$ & 0,00 & $3,87^{\text {n.s }}$ & 0,05 & $9,85^{* *}$ & 0,00 \\
\hline Classe (C) & 2 & $0,91^{\text {n.s }}$ & 0,40 & $4,01^{*}$ & 0,02 & $2,07^{\text {n.s }}$ & 0,13 & $4,12^{*}$ & 0,02 & $2,81^{\mathrm{n} . \mathrm{s}}$ & 0,06 \\
\hline$D \times A$ & 2 & $0,61^{\text {n.s }}$ & 0,54 & $2,12^{\text {n.s }}$ & 0,12 & $0,12^{\text {n.s }}$ & 0,88 & $0,28^{\text {n.s }}$ & 0,75 & $0,13^{\text {n.s }}$ & 0,87 \\
\hline $\mathrm{D} \times \mathrm{C}$ & 4 & $0,47^{\text {n.s }}$ & 0,75 & $2,25^{\text {n.s }}$ & 0,07 & $4,11^{* *}$ & 0,00 & $2,71^{* *}$ & 0,03 & $2,92^{* *}$ & 0,02 \\
\hline$A \times C$ & 2 & $0,13^{\text {n.s }}$ & 0,87 & $2,13^{\text {n.s }}$ & 0,12 & $0,06^{\text {n.s }}$ & 0,93 & $0,91^{\text {n.s }}$ & 0,40 & $0,17^{\text {n.s }}$ & 0,84 \\
\hline$D \times A \times C$ & 4 & $0,58^{\text {n.s }}$ & 0,67 & $1,61^{\text {n.s }}$ & 0,18 & $0,80^{\text {n.s }}$ & 0,52 & $0,27^{\mathrm{n} . \mathrm{s}}$ & 0,89 & $1,19^{n \cdot s}$ & 0,32 \\
\hline Resíduo & 54 & & & & & & & & & & \\
\hline
\end{tabular}

Em que: ns = não significativo a 5\% de significância; * = significativo a 5\% de significância; e ** = significativo a $1 \%$ de significância. 
posições $75 \%$ e $100 \%$, e no tocante à resistência à compressão paralela às fibras, na posição $75 \%$ do raio. Isso pode ter ocorrido devido à formação da madeira na época da ação da adubação ou a perda da influência da adubação (Figuras 3 e 4). Foi verificado que a massa específica e a resistência à compressão da madeira produzida das árvores provenientes das parcelas adubadas foram menores do que aquelas das madeiras produzidas nas parcelas não adubadas (Figuras 3 e 4).

A aplicação de fertilizantes após o desbaste aos cinco anos influenciou diretamente na produção de madeira de menor densidade aparente e resistência à compressão, em comparação com a madeira produzida nas parcelas não adubadas. Considerando que as parcelas adubadas produziram maior volume de madeira por unidade de área, pode-se, então, inferir que houve compensação da perda ocorrida na densidade aparente e resistência à compressão. Entretanto, isso só é verdade se o aumento do volume for mais do que proporcional à diminuição da densidade aparente e à resistência à compressão, o que possibilitaria o incremento da massa de matéria seca produzida por unidade de área. Land et al. (1983) e Barreiros et al. (2007) comprovaram essa observação. Tais resultados estão de acordo com os de Valeri et al. (1990) e Andrade et al. (1994). Entretanto, Wilkins e Kitaraha (1991), Vigneron et al. (1995), Berger et al. (2000) e Haselein et al. (2002) verificaram que a fertilização aumentou a densidade, MOE e MOR. Vital (1990) destacou a existência de resultados conflitantes a respeito do efeito da fertilização na densidade da madeira.

Obs.: Médias seguidas da mesma letra não diferem entre si, pelo teste de Tukey a 5\% de significância.

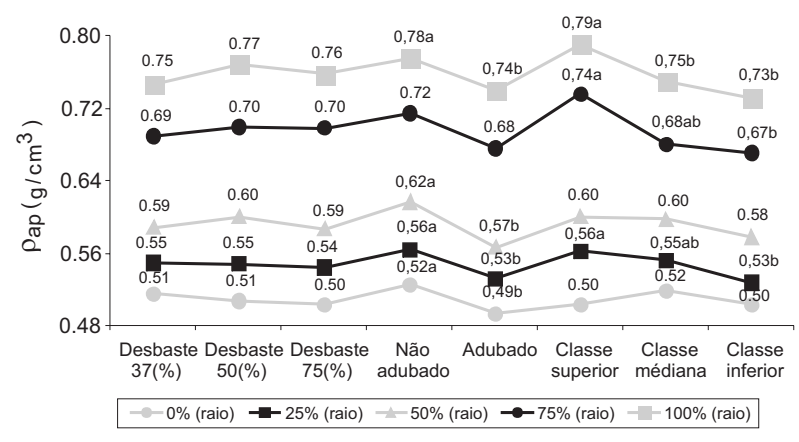

Figura 3 -Densidade parente $\left(\rho_{a p}\right)$ de $E$. grandis de 21 anos de idade.

Figure 3 - Specific density ( $\rho_{a p}$ ) of 21-year old E. Grandis.
As parcelas adubadas produziram madeira com menor densidade aparente e resistência à compressão ao longo do raio da tora da árvore. De acordo com Harris (1981) e Vital (1990), a diminuição da densidade aparente que as parcelas adubadas produziram foi devida à redução do comprimento das fibras e ao aumento na proporção de volume ocupado pelos vasos. Isso também contribui, muito provavelmente, para a diminuição da resistência da madeira.

Com relação às classes de DAP, verificaram-se que há diferenças significativas entre as classes, nas posições radiais 25 e $75 \%$, na densidade aparente e nas posições 25\% e 75\%, com relação à resistência (Figuras 3 e 4). A classe superior de diâmetros apresentou os maiores valores de massa específica e resistência e a classe inferior, os menores valores das duas propriedades.

Pode-se também observar, nas Figuras 3 e 4, que ocorreu aumento da densidade aparente e da resistência, no sentido da medula-casca, em todas as posições radiais. Esse incremento da densidade no sentido da medula para casca também foi observado nos trabalhos de Wilkes (1984), Wilkins (1990), Malan (1991), Malan e Hoon (1992) e Polli et al. (2006).

Neste estudo, foram feitas, também análises de correlações entre as variáveis $\tilde{\mathrm{n}}_{\mathrm{ap}}, \mathrm{f}_{\mathrm{c} 0}$ e a posição radial. As Figuras 5 a 7 mostram, graficamente, os modelos de regressões, que foram estatisticamente

Obs.: Médias seguidas da mesma letra não diferem entre si, pelo teste de Tukey a 5\% de significância.

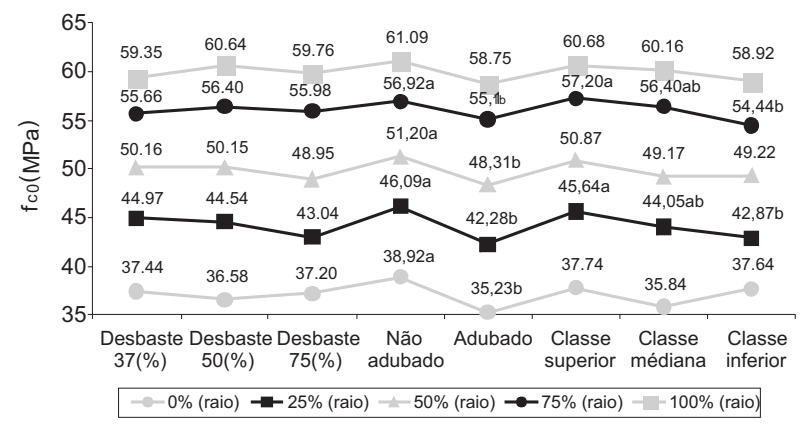

Figura 4-Resistência à compressão $\left(f_{\mathrm{c} 0}\right)$ de E. grandis de 21 anos de idade.

Figure 4-Compression strength $\left(f_{c 0}\right)$ of 21-year old E. Grandis. 


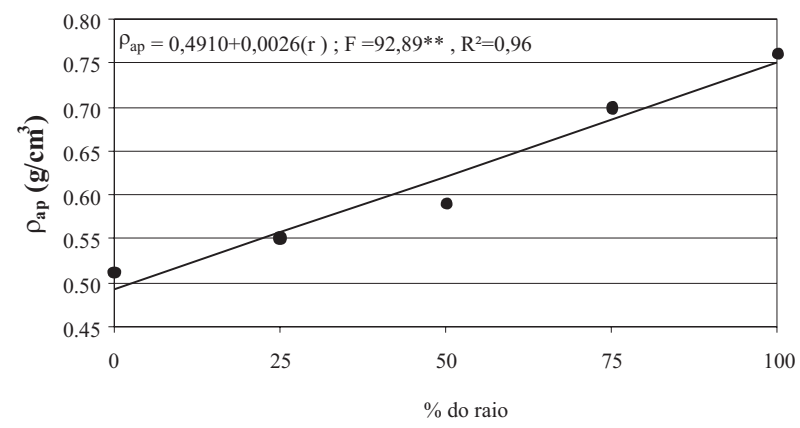

Figura 5 - Relação entre densidade aparente $\left(\rho_{a p}\right)$ e posição radial (r) na tora de E. grandis de 21 anos de idade.

Figure 5 - Relation between specific gravity $\left(\rho_{a p}\right)$ and the radial positions $(r)$ in the log of 21-year-old E. grandis.

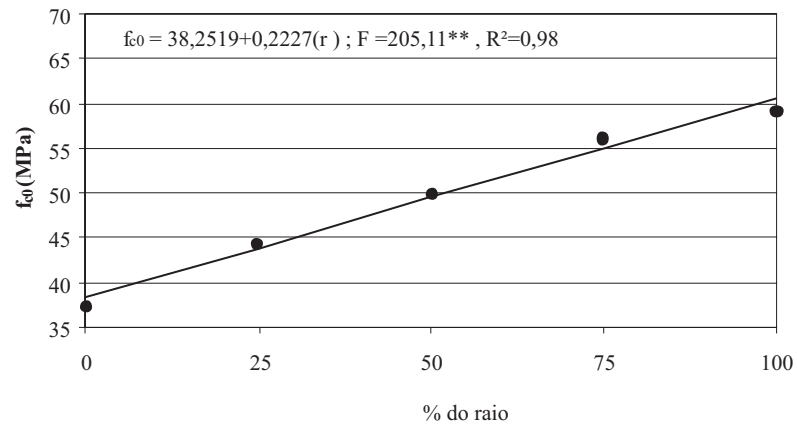

Figura 6 - Relação entre resistência à compressão $\left(f_{\mathrm{c} 0}\right)$ e posição radial (r) na tora de $E$. grandis de 21 anos de idade.

Figure 6 - Relation between compression strength $\left(f_{c 0}\right)$ and the radial positions $(r)$ in the log of 21-yearold E. grandis.

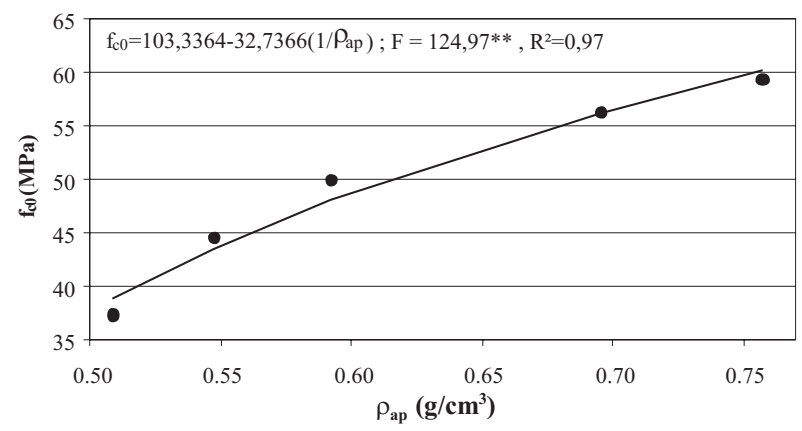

Figura 7 - Relação entre densidade aparente $\left(\rho_{a p}\right)$ e resistência à compressão $\left(f_{\mathrm{c} 0}\right)$ de $E$. grandis de 21 anos de idade.

Figure 7 - Relation between specific gravity $\left(\rho_{a p}\right)$ and compression strength $\left(f_{c 0}\right)$ of 21-year-old $E$. grandis. significativos. Forte dependência foi obtida entre posição radial e densidade aparente e posição radial e resistência à compressão (Figuras 5 e 6), o que permite estimar, com certa segurança, a densidade aparente e a resistência à compressão através da posição radial. O mesmo ocorreu quanto à relação positiva entre densidade aparente e resistência à compressão (Figura 7), permitindo estimar a resistência à compressão através dos valores obtidos de densidade aparente. Isso comprova a tendência de a densidade da madeira estar relacionada diretamente com a resistência, conforme indicaram Nascimento (1993), Scanavaca Junior e Garcia (2004) e Lima e Garcia (2005). Entretanto, Dias e Lahr (2004) observaram em madeiras tropicais que o modelo exponencial entre densidade aparente e resistência à compressão paralela às fibras foi o que apresentou melhor ajuste dessas propriedades.

\section{CONCLUSÕES}

Nas condições deste estudo pode-se concluir que:

A densidade aparente e a resistência à compressão da madeira não foram influenciadas pelas diferentes intensidades de desbaste.

A densidade aparente e a resistência à compressão da madeira foram influenciadas pelo fator adubo e classe de DAP, em quase todas as posições radiais.

A densidade aparente e a resistência à compressão são menores nas árvores oriundas de parcelas adubadas, em comparação com aquela de árvores procedentes de parcelas não adubadas.

A classe superior de diâmetros apresenta densidade aparente e resistência à compressão maiores do que a classe inferior.

A densidade aparente e a resistência à compressão aumentam significativamente, no sentido da medula para a casca.

Ocorreram forte dependência entre densidade aparente, resistência à compressão paralela às fibras e posição radial na tora.

\section{AGRADECIMENTOS}

Às empresas RIPASA S/A Celulose e Papel Ltda. e CAF Florestal, pelo fornecimento do material de estudo e pelo inestimável apoio logístico, o que propiciou o pleno desenvolvimento deste trabalho.

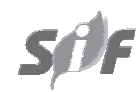

Revista Árvore, Viçosa-MG, v.34, n.3, p.551-559, 2010 


\section{REFERÊNCIAS}

ANDRADE, A.M. et al. Efeitos da fertilização mineral e da calagem do solo na produção e na qualidade da madeira de eucalipto. Revista Árvore, v.18, n.1. p.69-78, 1994.

\section{ASSOCIAÇÃO BRASILEIRA DE NORMAS} TÉCNICAS - ABNT. Projeto de estruturas de madeira: projeto NBR 7190/1997. Rio de Janeiro: 1997. 107p.

BARREIROS, R. M. et al. Modificações na produtividade e nas características físicas e químicas da madeira de Eucalyptus grandis causadas pela adubação com lodo de esgoto tratado. Revista Árvore, v.31,n.1. p.103-111, 2007.

BERGER, R. et al. Efeito do espaçamento e da fertilização sobre o crescimento e qualidade da madeira de um clone de Eucalyptus saligna SMITH. In: SIMPÓSIO LATINO-AMERICANO SOBRE MANEJO FLORESTAL, 1., 2000, Santa Maria. Anais... Santa Maria: UFSM, 2000. p. 209-236.

CRUZ, C. R.; LIMA, J. T.; MUNIZ, G. I. B. Variações dentro das árvores e entre clones das propriedades físicas e mecânicas de híbridos de Eucalyptus. Scientia Forestalis, n.64, p.33-47,2003.

DIAS, F. M.; LAHR, A. R. Estimativa de propriedades de resistência e rigidez da madeira através da densidade aparente. Scientia Forestalis, n.65, p.102-113, 2004.

HARRIS, J. M. Effect of rapid growth on wood processing. In: WORD CONGRESS, 17., Japan, 1981. Proceedings... Japan: IUFRO, 1981. p.117-125.

HASELEIN, C. R. et al. Propriedades de flexão estática da madeira e umidade e a $12 \%$ de umidade de um clone de Eucalyptus saligna Smith sob efeito do espaçamento e da adubação. Ciência Florestal, v.12, n.2, p.147-152, 2002.

LAND, S. B. et al. Genetic, site, and within-tree variation in specific gravity and moisture content of young sycamore trees. Tappi Journal, v.66, n.3, p.149-153, 1983.

LIMA, I. L.; GARCIA, J. N. Influência do desbaste em propriedades físicas e mecânicas da madeira de Eucalyptus grandis Hill ex-Maiden. Revista do Instituto Florertal, v.17, n.2, p.151-160, 2005.

Revista Árvore, Viçosa-MG, v.34, n.3, p.551-559, 2010
MALAN, F. S. Eucalypts improvement for lumber production. In: SEMINÁRIO INTERNACIONAL E UTILIZAÇÃO DAMADEIRADE EUCALIPTO PARA SERRARIA, São Paulo, 1995. Anais...Piracicaba: IPEF; IPT; IUFRO; ESALQ, 1995.p.1-19.

MALAN, F. S. Variation, association and inheritance of juvenile wood properties of Eucalyptus grandis Hill ex Maiden with special reference to the effect of rate of growth. South African Forestry Journal, n.157, p.16-23, 1991.

MALAN, F. S.; HOON, M. Effect of initial spacing and thinning on some wood properties of Eucalyptus grandis. South African

Forestry Journal, n.163, p.13-20, 1992.

NASCIMENTO, C. C. Variabilidade da densidade básica e de propriedades mecânicas de madeiras da Amazônia. 1993. 119f. Dissertação (Mestrado em Ciências Florestais) - Escola Superior de Agricultura “Luiz de Queiroz”, Piracicaba, 1993.

POLLI, H. Q. et al. Qualidade da madeira em clone de Eucalyptus grandis W. Hill ex Maiden submetido a desrama artificial. Revista Árvore, v.30, n. 4, p.557-566, 2006.

SAS. Institute Inc. SAS procedures guide. Version 8 (TSMO). Cary: 1999.

SCANAVACA JUNIOR, L.; GARCIA, J. N. Determinação das propriedades físicas e mecânicas da madeira de Eucalyptus urophylla. Scientia Forestalis, n.65, p.120-139, 2004.

VALERI, S. V. et al. Variação da densidade básica da madeira de Eucalyptus grandis Hill ex Maiden com a altura do caule e aplicação de fósforo e calcário dolomítico. In: CONGRESSO FLORESTAL BRASILEIRO, 6., 1990, Campos do Jordão. Anais... São Paulo: SBS/SBEF, 1990. p.746-750.

VIGNERON, P.; GERARD, J.; BOUVET, J. M. Relationship between basic density and growth in a fertilization trial with clones of Eucalyptus hybrids in Congo. In: POTTS, B. M. et al. EUCALYPT PLANTATIONS: IMPROVING FIBRE YIELDAND QUALITY, 1995, Hobart. Proceedings... Sandy Bay: CRCTHF/IUFRO, 1995. v.1.p.68-72.

VITAL, B. R. Reflexos da fertilização mineral na qualidade e na utilização da madeira. In: BARROS, N. F.; NOVAIS, R. F. Relação solo-eucalipto. Viçosa, MG: Folha de Viçosa, 1990. p.323-330.

Revista Árvore, Viçosa-MG, v.34, n.3, p.551-559, 2010 
WILKES, J. The influence of rate of growth on the density and heartwood extractives content in eucalypt species. Wood Science and Technology, v.18, n.2, p.113-20, 1984.

WILKINS, A. P. Influence of Silvicultural treatments on growth and wood density of Eucalyptus grandis grown on a previous pasture site. Australian Forestry, v.53, n.3, p.168-172, 1990.
WILKINS, A. P.; HORNE, R. Wood-density variation of young plantation grown Eucalyptus grandis in response to silvicultural treatments. Forest Ecology and Management, Amsterdam, v.40, n.1/2, p.39-50, 1991.

WILKINS, A. P.; KITAHARA, R. Silvicultural treatments and associated growth rates, growth strains and wood properties in 12.5-year-old Eucalyptus grandis. Australian Forestry, v.54, n.1/2, p.99-104, 1991. 